How to reference this article Palmarini, L. (2019). Sul manoscritto testamentario di Bartolomeo Berrecci: uno sguardo storico-linguistico. Italica Wratislaviensia, 10(1), 309-329.

DOI: http://dx.doi.org/10.15804/IW.2019.10.1.13

\author{
Luca Palmarini \\ Uniwersytet Jagielloński \\ luca.palmarini@uj.edu.pl, ORCID: 0000-0002-4223-8290
}

\title{
SUL MANOSCRITTO TESTAMENTARIO DI BARTOLOMEO BERRECCI: UNO SGUARDO STORICO-LINGUISTICO
}

\author{
ON THE TESTAMENTARY MANUSCRIPT \\ OF BARTOLOMEO BERRECCI: \\ A HISTORICAL AND LINGUISTIC VIEW
}

\begin{abstract}
The following article presents the analysis of the last will of the most famous Renaissance sculptor and architect in Poland: the Tuscan Bartolomeo Berrecci, creator of the Sigismund Chapel in the Wawel Cathedral in Kraków. After a brief historical-cultural introduction where the artist's arrival in Poland and his function at the court of King Sigismund I the Old are presented, the transcription of the artist's testament, written by his own hand in the Italian language and recorded in the archives of the ancient city of Kazimierz, is included, followed by an in-depth analysis. At first, the analysis will concentrate on content and structure: the characters and properties mentioned in the document will be presented along with the document's form. Subsequently, morphological and terminological analyses will be carried out, which will confirm the use of the Florentine language of the late-fifteenth and early-sixteenth centuries, as well as the presence of specific terms related to the Polish reality in which the artist lived for almost 20 years, giving the document historical and linguistic value, in addition to classic values that are spiritual and notarial.
\end{abstract}

Keywords: Italian in XVI century, Bartolomeo Berrecci, Testament of Bartolomeo Berrecci, Renaissance art, Renaissance in Kraków 
Dresso l'Archivio Nazionale Polacco (Archiwum Narodowe), sezio1 ne di Cracovia, negli Acta d. scabinorum civitatis Kazimirie pos exustionem pretorii per manus Bartolomei Cromeri notarii comperata, volume 380 della Dokumentacja aktowa, è conservato il testamento dell'architetto e scultore italiano Bartolomeo Berrecci di Pontassieve (1480 c.-1537). Si tratta di uno scritto di due pagine, precisamente ai numeri 364 e 365, vergato a mano e firmato dall'artista toscano che, nel 1536, ovvero un anno prima della sua morte, decise di scrivere a chi avrebbe lasciato i suoi averi. Il volume degli Acta, in cui è compreso questo documento, raccoglie la documentazione dal 26 VI 1528 al 23 XII 1541 riguardante la città di residenza di Berrecci, ovvero Kazimierz (Casimiria). Di conseguenza, sempre negli Acta sono presenti altri documenti ufficiali riguardanti la vita dell'artista di Pontassieve in Polonia, ma il testamento, scritto più intimo e personale, è l'unico di essi redatto in lingua italiana.

\section{BARTOLOMEO BERRECCI A CRACOVIA (1516-1537)}

Berrecci passò buona parte della sua vita a Cracovia, città in cui trovò la sua consacrazione ad artista di alto rango. Il suo operato si è rivelato fondamentale nel confermare il modello rinascimentale toscano-romano al di fuori dei confini naturali della penisola italiana. Sulla produzione artistica di Berrecci si è scritto molto, soprattutto da parte polacca; ciò è avvenuto per ovvi motivi, considerata la sua lunga permanenza in questo paese (1516-1537) e la produzione artistica di maggior rilievo qui realizzata. L'apice dell'interesse scientifico verso questa personalità si è avuto a cavallo tra i secoli XIX e XX, quando, anche grazie alle spinte risorgimentali polacche, l'attenzione per l'arte nazionale ebbe un notevole sviluppo. Gli studi sugli artisti rinascimentali italiani in Polonia sono poi continuati con grande fervore per tutto il Novecento fino ai giorni nostri. Da parte italiana, per avere una monografia dedicata all'architetto toscano si è dovuta invece attendere l'opera Bartolomeo Berrecci da Pontassieve. Un genio del Rinascimento tra arte e filosofia (Cappelletti, 2011). 
In questa sede si ritiene opportuno presentare brevemente l'attività artistica di Berrecci, citando alcuni particolari della sua vita privata che possono poi essere ricollegati allo scritto testamentario qui analizzato.

Si presume che Berrecci sia arrivato a Cracovia nel 1516 via Ungheria, chiamato dal re Sigismondo I al fine di continuare il lavoro dell'architetto di corte Francesco Fiorentino, deceduto prematuramente. A Cracovia l'artista toscano si occupa soprattutto della realizzazione della Cappella di Sigismondo (1519-1533), monumento funebre oggi considerato un capolavoro dell'arte rinascimentale, all'interno della cui cupola l'autore fa scolpire la scritta: "Bartholo Florentino Opifice". Dal 1527 in poi lavora alla struttura del castello di Wawel (portali e finestre) e alla tomba in marmo dell'arcivescovo Tomicki. La figura di Berrecci viene indubbiamente ritenuta cruciale per lo sviluppo del Rinascimento in Polonia:

Berrecci jest [...] najwybitniejszym przedstawicielem nurtu włoskiego w polskiej sztuce renesansu. Jako architekt i jako rzeźbiarz wprowadza do nas włoskie rozwiązania, nadając im indywidualne piętno. Styl jego wywodzie się z tradycji florenckiego Quattrocenta, ale liczne nawiązania do sztuki początku XVI wieku w Rzymie świadczą o jego pobycie w tym mieście. (Kozakiewicz \& Kozakiewicz, 1976, p. 14) ${ }^{1}$

Due date da segnalare sono il 21 marzo del 1528, quando viene nominato cittadino di Casimiria, e il 17 aprile del 1533, giorno in cui ottiene l'incarico di consigliere cittadino (tawnik). Interessanti, nel contesto di questa ricerca, sono anche i legami tra gli artisti italiani presenti a Cracovia che si intensificano al punto da creare una fitta rete di contatti e amicizie che arriverà a prendere il nome di familia e di cui Berrecci sarà una della figure di spicco. Molto discusse sono state anche le circostanze della sua morte: al tempo a Cracovia la concorrenza per gli appalti riguardanti le opere artistiche ed edilizie era molto accesa

1 "Berrecci è $[\ldots]$ il più eminente rappresentante della corrente italiana nell'arte rinascimentale polacca. Come architetto e scultore introduce da noi le soluzioni italiane, dando loro un'impronta individuale. Il suo stile nasce dalla tradizione del Quattrocento fiorentino, ma i numerosi legami con l'arte dell'inizio del XVI secolo a Roma confermano il suo soggiorno in questa città" (trad. mia). 
e spesso portava a regolamenti di conti tra gli stessi artisti. Anche la rivalità tra $\mathrm{i}$ costruttori italiani presenti nella capitale polacca di allora era molto agguerrita (Pelc, 2000, p. 190) e, probabilmente, è proprio per questo motivo che nell'estate del $1537^{2}$ l'artista viene accoltellato da un altro italiano: "Bartholomeus Berreci Florentinus [...] interfectus fraudolenter per unum italum ex invidia, in circulo Cracoviensis" (Mossakowski, 2007, p. 303). Negli atti cittadini, però, non si è conservato alcun documento riguardante un eventuale processo a carico di un ipotetico assassino.

Si è concordi sul fatto che la morte prematura del toscano abbia posto un brusco freno allo sviluppo artistico polacco che adattava il metodo italiano alle soluzioni tardogotiche locali, lasciando invece spazio a un modello artistico più pluralista (Fabiański, 2000, p. 39).

\section{LA TRASCRIZIONE DEL TESTAMENTO}

Una prima trascrizione del testamento manoscritto si ritrova negli Sprawozdania Komisji do Badania Historii Sztuki w Polsce del 1891 (Akademia Umiejętności, 1891, libro IV, pp. XXVI e XXVII), ma questo lavoro non è scevro da molti errori di trascrizione e comprensione, nonché dalla mancanza di accenti e della segnalazione del completamento delle parole abbreviate, così come da molte informazioni storiche. Karol Estreicher, invece, che presenta a grandi linee il testamento del toscano, ne propone una traduzione libera in lingua polacca (Estreicher, 1972, pp. 51-52). Al fine di rendere più precisa la successiva analisi si propone in questa sede una nuova trascrizione, ispirata ai criteri filologico-linguistici ${ }^{3}$, realizzata dall'autore dell'articolo. Ecco, dunque, il testamento di Bartolomeo Berrecci:

2 In "feria secunda post Francisci" Berrecci viene citato già come defunto (Acta d. Scabinorum, 1536, nr 380, p. 201).

3 La mia trascrizione conserva al massimo la punteggiatura dell'originale, comprese le virgole e i due punti, e l'uso delle maiuscole nonostante ciò discosti tanto dall'uso moderno. Integro solo con il corsivo le parole che nel testo originale sono abbreviate e aggiungo gli accenti. 
In nomine domini nostri Hiesu Christi: io Bartholo di Lucca Berrecci fiorintino habitante et citadino in Casimiria: sano di la mente et del corpo cognoscendo non essere cosa più certa che la morte, né cosa più incerta de l'hora sua: et perché tutto sta nella voluntà di nostro signor Hiesu Christo et io timendo di non potere al extremo acconciare le cose mie: ho fatto et scripto questo testamento di mia propria mano et serà sigillato con il mio sigillo et questa è la mia ultima voluntà anulando ogni altra scripta che io havesse facta per il passato et in questo lasso et prego per veri et ligitimi testamentari ovvero executori di questo testamento Messer Casparo Gucci fiorentino uno, et Malcher viceprocuratore alias podrenzi dopo la morte mia possano procedere secundo di qui di sobto se trovarà scripto, et si l'uno ci fussi et l'altro non commetto et do quella libertà a quel uno solo, che si fussino ambodua et si li sopra scripti dua non si accordassero prego che per il terzo sia ne Justo Ludovico et habiano integra auctorità a procedere come veri et legitimi executori. In prima raccomando l'anima a nostro Signore Jesu Christo humilmente pregandolo che per la sua pietà et infinita misericordia se degni perdonarmi li miei peccati et l'anima mia conducere a loco di salvazione: item il corpo mio lasso sia sepellito nela chiesa del Corpus Domini et per la sepultura si spenda fiorini trenta: Item elemosine fiorini deci dove meglio parerà alli executori et a mia moglie: In prima consegno sopra ogni mio bene mobile et immobile fiorini quattrocento di moneta alla mia chara moglie Dorothea, li quali gli promessi tricento quando la sposai et cento ne gli aiungo et tamen voglio che sia contenta: Item la quarta parte di massaricie di casa stagni et rame et altre cose domestiche. Item al presente mi trovo quaranta octo cocchiare di argento, voglio che dodici siano sue di Dorothea: Item voglio che habia a vita sua non si volendo remaritare habitazione, quanto se richiede in casa dove hoggi io habito, ovvero nella casa che fu di Venzko, dove meglio parerà alli soprascripti tutori purché l'habi a sufficienzia et pacifica habitazione et questo basti alla mia dilecta moglie: Item voglio che sia di Catherina et Anna mie figliole la casa dove al presente habito, un'altra casa presso [365] alla Judaica drieto aureco un'altra piacza in sul cantone de la piacza a riscontro a valenti beccaijo: un'altra casa in sul cantone de la via de Viliska, in 
piacza due altre in dicta via infra Agnesa Colassina et urbanchova, una casa et due piacze: 1'altra dove è lo fornaro infra nogajska Sluska: Item dua pannicidi, uno mio libero sobto la stufecta del pretorio et l'altro a riscontro che ni ho sopra trenta marche prestate in contante: item la casa de Anna caffeczanka in sul cantone de la piacza in su la quale è la resignatione de la mezza casa per ducento fiorini; tucte queste et più se ne comprasse per lo avenire, voglio che siano di Catherina et Anna, mie due figliole tanto con pacti che non si trovasse dinari contanti da sadisfare alla mia moglie Dorothea, voglio che dicte due figliole Catherina et Anna in termine de un mezzo anno pagano fiorini tricento per la dote che ni ho facta a mia mogliere. Gli altri cento fiorini gline consegno sopra alla mia fornace fuori di Casmira: Item a Catherina et Anna octo bicchieri d'argento et vintiquattro cocchiare d'argento et mezze le massaricie di casa stagni, rami et panni di lino et lane. Seguita tucti li mei debitori come se trova scripto in su lo mio registro voglio che Catherina et Anna habiano integra auctorità a riscoterli et di quelli pagare se io lassasse qualche debito tanto che ogniuno sia sadisfacto: del resto lasso ogni cosa a Catherina et Anna mie figliole eccepto che il prato fuori di Casmira, et piscina et fornace con ogni altro edificio in quel loco, quello mi reservo in mio arbitrio quanto mi piacerà con ogni iure insieme con dudici cocchiare d'argento et una coppa doppia d'argento indorata dentro et fuori et lo mio sigillo d'oro, cioè anello et la quarta parte di massaricie di casa; tucti queste cose mi reservo darli et consignarli a mio piacere con lo adiuto del altissimo: Item reservandomi de ogni et qualunque cosa libera et integra auctorità mentre che so vivo, di ciocchè di sopra è scripto, vendere, impignare, dare donare, a mio beneplacito, convertire come vero patrono di tucte quelle cose, che in mia libertà me li reservo et questo testamento acconciarlo levare et ponere come a me parerà meglio in finch'io vivo. Tanto non facendo altro testamento voglio che questo sia valido et che la mia moglie Dorothea et Catherina et Anna siano contente queste cose da me ordinate et di mia propria mano scripte et per più fede serà sigillato col mio proprio sigillo et dato asservo in pretorio ne la casa de iurati ovvero di consuli, scripto hoggi alli XXIII di gennaro M D XXXVI

Io Bartholo soprascripto scripsi mano propria 


\section{OSSERVAZIONI SUL TESTAMENTO}

Il testamento viene scritto e firmato il 23 gennaio 1536. Uno dei motivi della decisione della sua stesura, oltre a una possibile età avanzata, lo si può identificare nella rapida diffusione che in quel periodo in Europa stava avendo l'epidemia della peste (Łuszczkiewicz, 1879, p. 33), ma, tenendo in considerazione la morte violenta cui fu soggetto il toscano, è plausibile l'ipotesi che Berrecci avesse ricevuto delle minacce di morte. Nelle mie ricerche d'archivio, infatti, sono venuto a conoscenza del fatto che poco dopo anche la moglie fa testamento (Acta d. Scabinorum, 1536, p. 169): "Dorothea Czarnowoysczanka uxor Circumspecti Batholomei Itali Lapicidi" decide di lasciare in caso di morte "omnia bona sua mobilia et immobilia" al marito. Il testamento di Dorota porta la data del "sabbato di sancte Agate", ovvero il 5 febbraio 1536. La breve distanza temporale che intercorre tra la stesura dei due atti notarili può indurci a credere che i coniugi Berrecci percepissero una situazione di pericolo.

Il testo è redatto in prima persona, pratica allora non sempre comune. Per quanto riguarda la lingua utilizzata, si tratta indubbiamente del fiorentino di fine Quattrocento, con elementi del primo Cinquecento, ma anche interessanti ingerenze e sorprese di cui si troverà conferma in seguito.

Dal XII secolo in poi il testamento aveva cessato di essere solo un atto di diritto privato che aveva come unico scopo la regolamentazione della successione dei beni. Questo tipo di documento ottemperava a una duplice funzione: era infatti diventato anche un atto religioso praticamente imposto dalla Chiesa che minacciava di scomunica chi non lo avesse sottoscritto. Con tale dichiarazione il fedele si riscattava dai suoi peccati compiendo un atto pubblico, mentre la Chiesa aveva uno strumento di controllo del peccatore e allo stesso tempo una sicura fonte di guadagno, in quanto dall'eredità veniva prelevata una decima.

Così sembra presentarsi anche il testamento di Berrecci che, però, a differenza di altri documenti dell'epoca, come p. es. nel caso del testamento di Leonardo ${ }^{4}$, non si dilunga in lodi a diversi santi, bensì si

4 Il testamento di Leonardo, infatti, recita: "Primeramente el racomanda l'anima sua ad nostro Signore Messer Domine Dio, alla gloriosa Virgine Maria, a Monsignore 
rivolge solamente al Signore Gesù. La frase d'esordio del testo di Berrecci "In nomine domini nostri Hiesu Christi" è laconica ed è in latino. È interessante sottolineare che la formula si presenta identica nella struttura e nella morfologia a quella riportata all'inizio delle tesi di Martin Lutero, ma potrebbe trattarsi solamente di un caso. Anche Estreicher nota un certo atteggiamento di freddezza di Berrecci nei confronti della Chiesa (1972, p. 54).

All'inizio del testamento lo scultore si presenta così: "Bartholo di Lucca Berrecci fiorintino habitante et citadino in Casimiria". Il "di Lucca" si riferisce chiaramente al padre con il significato di "figlio di Luca". Riguardo alla definizione "fiorintino", oppure al più frequentemente riscontrato latino "Florentinus" (o ancora il latineggiante Florentino), che spesso accompagnano il nome Bartolomeo in molti documenti, si può essere concordi sul fatto che gli stranieri erano soliti aggiungere un appellativo che si riferiva alla città più grande situata nelle vicinanze del paese in cui erano nati, in quanto più conosciuta, oppure quello della città in cui aveva sede la diocesi da cui dipendevano. Questa fu anche la scelta generale di Berrecci. L'appellativo riguardante la precisa provenienza da Pontassieve lo si ritrova invece in almeno due documenti: il primo è una causa ecclesiastica riguardante il figlio Sebastiano, in cui Berrecci si dichiara pronto a pagare alla madre i costi della sua tutela e formazione. Qui l'artista è chiamato "Bartholomeus de Ponte Seve, laicus diocesi florentine, Italus Lapicida" (Acta officialia Archidiecezji Krakowskiej 57, 1520, pp. 96v e 97r). Nel testamento di uno degli scultori reali, Andrea de Nicolo, morto nel 1523, il suo esecutore "Raphael Florentinus lapicida" assolve dagli obblighi "Bartholomeum de Ponte Asseve florentinum lapicidam" (ivi, 1521, pp. 525v e 526r). In altri documenti ufficiali spesso è presente la dizione latina "Bartholomeus italicus" o "italus", a volte con la variante "Bartholomaeus" (Ferenc, 1952, pp. 54 e 84), o ancora, più raramente, tale appellativo viene reso in lingua tedesca, "Bartosch der Wall" (Akademia Umiejętności, 1906, p. 4).

Sancto Michele, e a tutti li beati Angeli Santi e Sante del Paradiso" (Uzielli, 1872, p. 212). 
Continuando la lettura del testamento di Berrecci, si osserva che il suo latore si dichiara abitante e cittadino di Casimiria. Il toponimo è riportato nel testo ben tre volte: la prima nella dizione completa di "Casimiria", in seguito con lo storpiamento "Casmira". Dopo aver dichiarato la propria sanità mentale, l'autore dello scritto riporta la frase: "cognoscendo non essere cosa più certa che la morte né cosa più incerta de l'hora sua". Si tratta di una massima assai popolare nei testamenti dell'epoca, in quanto in forma assai simile si riscontra p. es. nel citato testamento di Leonardo, dove leggiamo: "el qual considerando la certezza della morte e l'incertezza dell'ora di quella" (Uzielli, 1872, p. 202). Tale formula mantiene la sua popolarità negli ambienti notarili italiani, seppur con lievi cambiamenti di forma, fino al XVIII secolo, dove ancora la si ritrova in sud Italia (Gaudioso, 1986, p. 30). Berrecci chiede di porre il suo sigillo, dichiarando: "la mia ultima voluntà anulando ogni altra scripta che io havesse facta per il passato". In effetti, in precedenza il toscano aveva stilato altri testamenti tra cui uno nel quale aveva deciso che in caso di morte metà del suo patrimonio sarebbe andata alla prima moglie.

In seguito l'artista cita gli esecutori materiali del suo testamento: "Casparo Gucci fiorentino" e "Malcher viceprocuratore", più un terzo, "Justo Ludovico", in caso di impossibilità dei primi due di mettersi d'accordo. Gasparo o Casparo Gucci era un ricco mercante fiorentino polonizzato, proveniente da una ricchissima famiglia insediatasi a Cracovia, dalla quale aveva ottenuto la cittadinanza nel 1534: "Caspar Guczi de Florentia mercator, ius suscepit civile" (Ptaśnik, 1909, p. 3). Nel testamento Berrecci sottolinea l'appartenenza di Gucci alla sfera culturale fiorentina, quasi a volerne confermare l'italianità e l'importante ruolo che svolgeva la familia. "Malcher viceprocuratore" è invece Malcher Czyrzowski (Czirzowski), "viceprocurator castri cracoviensis", formatosi a Padova, di cui si trovano alcune tracce nelle fatture riguardanti i lavori al castello del Wawel (Ferenc, 1952, p. 1), ma anche in un inventario post mortem del 14 luglio 1542, dove, oltre ai ricchi arredi d'uso quotidiano, sono stati rinvenuti otto libri di carattere religioso e giuridico, tra cui Moriae encomium (Szelińska, 1990, pp. 44-45). Il terzo esecutore testamentario, scelto come eventuale paciere, è Justus Ludovicus 
Decius (Jost Ludwik Decjusz), mercante cracoviano di origine alsaziana, diplomatico, segretario di Sigismondo I e amico intimo di Berrecci. Nel 1535, proprio su iniziativa di Decius, a Wola Chełmska (oggi Wola Justowska) viene costruita una villa suburbana su modello di quelle italiane che, già da subito dopo la sua realizzazione, riceve l'appellativo di "castellum elegantissimum" (Pawlicki, 2015, p. 24). Molti storici dell'arte sono concordi nell'attribuire a Berrecci e altri italiani tra cui Giovanni Cini, Bernardino De Gianotis, Filippo di Fiesole - alla familia insomma - la prima realizzazione dell'opera ${ }^{5}$. Decius, inoltre, aveva stretto una grande amicizia con Erasmo da Rotterdam e si era recato in visita personale da Martin Lutero. Inoltre, parimenti a Malcher, il ricco mercante possedeva nella sua biblioteca una pubblicazione di Erasmo. Da qui, ricollegandoci anche alla formula di esordio del suo testamento, si potrebbe immaginare che Berrecci, al pari dei suoi amici, fosse rimasto affascinato dagli scritti critici verso la Chiesa di Roma e dalla Riforma. Il testamento dimostra inoltre come l'architetto italiano fosse profondamente inserito nel tessuto dell'alta società polacca di allora.

Dopo le lodi al Signore, Berrecci continua le sue volontà con la richiesta di essere sepolto nella chiesa del Corpus Domini, lasciando un'offerta e disponendo la quota per la sepoltura.

Molto forte è l'attaccamento dell'artista di Pontassieve alla consorte: prima definisce Dorothea "cara moglie", per poi passare a "mia dilecta moglie", esprimendo anche il desiderio "voglio che sia contenta". Il sentimento d'affetto viene espresso anche verso le due figlie Anna e Catherina tramite l'utilizzo del vezzeggiativo "figliole". Infine Berrecci si prodiga nel sottolineare ulteriormente il suo augurio: "che la mia moglie Dorothea et Catherina et Anna siano contente queste cose da me ordinate". Si coglie qui un affetto sincero verso le tre donne, mentre non vi è menzione del primo figlio Sebastiano.

5 A conferma dell'esistenza di questo sistema di stretti contatti si ha il testamento di Giovanni Cini da Siena scritto nel 1529, prima della sua partenza dalla Polonia per 1'Italia, per un periodo di due anni, in cui, in caso di morte, tutto il suo patrimonio sarebbe passato agli artisti Bartolomeo Berrecci e Bernardo da Roma (Olkiewicz, 1979, p. 176). 
Come si evince dal testamento, il "lapicida" possedeva alcuni immobili tra Cracovia e Casimiria. Di uno di essi scrive: "in casa dove oggi habito ovvero nella casa che fu di Venzko". Ė suo desiderio lasciarlo alle figlie. Con ogni probabilità si tratta della casa allora presso la piazza del Mercato di Casimiria; se ne trova traccia negli atti cittadini (Acta d. Scabinorum..., 1535, p. 158), dove l'immobile viene localizzato "in circulo Maiori". Si ha anche la conferma che Anna aveva ricevuto l'eredità, in quanto nel 1543 la figlia "Bartholomaei Itali Florentini Lapicidae civis kazmiriensis" (Krasnowolski, 1992, p. 83), moglie di Jan Południe, decide di venderla a un altro consigliere cittadino. Successivamente vengono citati altri immobili lasciati alle figlie nelle vie Viliska (oggi Krakowska) e Judaica (oggi Józefa), uno vicino al "fornaro" (la fornace), uno "sobto la stufecta" (il forno di ceramica) del pretorio. Esistono ulteriori proprietà riguardo alle quali il toscano afferma: "quello mi reservo in mio arbitrio quanto mi piacerà con ogni iure". Si tratta di una fornace, di un prato e di una "piscina" (vasca) situate fuori Casimiria. Probabilmente sono alcuni dei lasciti al figlio Sebastiano; infatti, negli anni a venire si registra da parte di quest'ultimo proprio la vendita di una fornace (voce 'Berrecci Bartolomeo' in Polski Stownik Biograficzny, PAU, 1935, p. 468).

\section{ALCUNE OSSERVAZIONI SULLA MORFOLOGIA E SULLA TERMINOLOGIA}

Il testamento si presenta conforme alle soluzioni fiorentine della fine del Quattrocento-inizio Cinquecento. Berrecci lascia l'Italia per cercare fortuna all'estero tra la prima e la seconda decade del sedicesimo secolo, di conseguenza non viene coinvolto dai cambiamenti linguistici che si stanno imponendo nella penisola italiana con il lavoro di Pietro Bembo. L'architetto toscano offre i suoi servigi a corti lontane, anche se,

${ }^{6}$ Oggi la piazza del mercato di Kazimierz si chiama Plac Wolnica e si presenta assai ridimensionata rispetto alla sua struttura rinascimentale. La casa di Berrecci si trovava allora all'angolo della piazza con la via Platea Salis, via Wielicka, appunto; oggi corrisponde al numero 46 di via Krakowska. 
però, queste presentano forti legami con la penisola italiana. In Polonia il culto per le humanae literae era già sviluppato con la presenza nel Quattrocento di umanisti come Francesco Filelfo e Filippo Buonaccorsi, fenomeno intensificatosi a cavallo dei secoli XV e XVI dalla disputa tra Marsilio Ficino e lo stesso Buonaccorsi riguardo al rapporto tra 1'anima e il corpo (Cappelletti, 2011, p. 95). L'artista toscano partecipa ai dibattiti, frequenta il circolo culturale cracoviense Sodalitas Vistulana ${ }^{7}$, subendo il fascino della vacatio animae di Ficino, dottrina filosofica che si rifletterà poi nelle sue sculture. Il nostro scultore presentava, dunque, una preparazione culturale di alto livello, lo si può considerare colto, ma non disponendo di una sua produzione letteraria, non lo possiamo trattare al pari di uno scrittore. Inoltre, non si hanno conferme di un suo eventuale interesse riguardo alla questione della lingua.

La lingua di Berrecci è un fiorentino d'uso, semplice e dinamico, così come deve essere, in quanto in questo caso si tratta di un documento di pratico utilizzo, scritto in una lingua abbastanza diretta, dove l'uso dei tempi e dei modi non si rivela essere complicato. L'autore nelle sue indicazioni esprime indirettamente il desiderio di essere compreso, come un documento di tal tipo giustamente richiede. Sembra essere qui presente una componente formulare, cioè uno schema tipologico, abbastanza riconoscibile, con i tre momenti della salutatio, dell'exordium e della conclusio, quasi lontanamente a ricalcare un fenomeno allora assai diffuso anche nella tipologia delle lettere dei mercanti. La struttura del testamento sembra, dunque, voler in parte rispettare quel cliché di documenti scritti dalla classe mercantile fiorentina del pieno Quattrocento i cui rappresentanti sono scaltri (Berrecci non è solo artista ma anche proprietario di case, fornaci, cave e altro ${ }^{8}$ ) e bene inseriti nelle strutture sociali (le persone citate sembrano essere suoi buoni conoscenti). Al tempo stesso l'artista svolge il ruolo di padre di famiglia ed educatore,

7 Il circolo culturale Soliditas vistulana fu fondato nel 1489 dall'umanista tedesco Konrad Celtis.

8 Per esempio nella Mała Encyklopedia Krakowa (Adamczewski, 1997, p. 34) oltre che artista Berrecci viene definito 'imprenditore'. 
elemento spesso sottolineato negli scritti dei commercianti toscani colti e semicolti della seconda metà del Quattrocento.

L'interpunzione del documento conserva la consuetudine quattrocentesca dei testi volgari. Infatti, le pause intermedie sono segnalate dai due punti (qui preferiti alla barra). Anche la grafia ${ }^{9}$ si presenta di impronta tardo quattrocentesca e dei primi del Cinquecento: l'affricata alveolare è resa con $c z$ : piacza; la nasale palatale, invece, viene riportata con la grafia gn: ogniuno, resignatione, cognoscendo.

Dal punto di vista morfologico vengono conservati alcuni nessi come $k s$ riportato con la lettera $x$ etimologica: executori, per 'esecutori', extremo per 'estremo', oppure il nesso ct: facta per 'fatta', auctorità per 'autorità', octo per 'otto', dilecta per 'diletta', dicta per 'detta', stufecta per 'stufetta', tucte per 'tutte', pacti per 'patti', dicte per 'dette', sadisfacto per 'soddisfatto' e quelli con il nesso pt: scripto per 'scritto', ecepto per 'eccetto', soprascripto per 'soprascritto'. Presente è anche il nesso $b t$ con sobto per 'sotto'. Si riscontra, inoltre, la grafia latineggiante nell'uso della $h$ : habitante per 'abitante', habitazione per 'abitazione', (io) habito per 'abito', habia per 'abbia' e habiano per 'abbiano', hora per 'ora', humilmente per 'umilmente' e infine hoggi per 'oggi'. Gli influssi latineggianti sono visibili nell'uscita in -tione del termine resignatione, ma anche in quello presentato con la $z$, salvazione, così come, in parte, nell'uscita -zia nel caso del termine sufficienzia. Latineggiante (e allo stesso tempo dialettale) è anche la monottongazione riscontrata nel verbo riscotere, invece di 'riscuotere'. La svolta bembiana, invece, propenderà per una dittongazione toscana trecentesca, come $\mathrm{p}$. es. nel caso di cuore, al posto della monottongazione che era corrente nel toscano quattrocentesco e che Berrecci adopera secondo la sua sensibilità fiorentina. Un ulteriore suggerimento di elementi del fiorentino vivo dell'inizio del Cinquecento è la presenza del numerale $d u a$ e del composto ambedua. Con la lezione bembiana nella lingua italiana si andrà a una selezione delle forme per cui prevarrà due e non dua, che era pur ben presente nel fiorentino cinquecentesco. Ne troviamo conferma ad

9 Per una maggiore visibilità, i termini estrapolati dal testo per l'analisi linguistica verranno riportati in corsivo. 
esempio nel trattato Giuochi mathematici di Niccolò da Filicaia (1516, pp. 47r, 47v, 124r), dove la forma dua si alterna a due. Chiaramente si ricorda che il testamento qui analizzato è uno scritto assai breve, quindi occorrenze di tal tipo si presentano in singoli casi, è difficile affermare se Berrecci usasse solo dua oppure lo alternasse ad altre forme. Allo stesso modo lo scultore toscano riporta deci invece di dieci. La medesima situazione si osserva nelle lettere dell'Ariosto antecedenti al 1516 (Marazzini, 2002, p. 275); dopo quella data, infatti, Ariosto si sarebbe adeguato ai dettami del Bembo, proponendo, tra gli altri, dieci al posto del precedentemente utilizzato deci. Oscillazioni presenti in Berrecci sono invece quella tra le forme Hiesu e Jesu, citate in precedenza, dove Berrecci opta due volte per la prima e una per la seconda, e dodici e $d u$ dici, presenti nel fiorentino del XV secolo.

La forma cognoscere presenta una grafia latineggiante, anche se probabilmente già allora possedeva una pronuncia locale. Il latino item va oltre l'utilizzo dei latinismi dell'epoca, in quanto trattasi di un prestito non integrato che per secoli riguardava l'ambiente notarile: "Item (ant.) parimenti, ugualmente, altresì; è frequente nel linguaggio notarile antico, specialmente nelle formule dei testamenti, come voce di ripresa in un'elencazione" (v. voce 'item' in Garzanti Linguistica on line). Lo stesso vale per tamen. Altri termini latini sono, oltre alla lode iniziale a Gesù, anche iure, da cui si riscontra la forma latinizzante iurati, e l'interessante pannicidi, prestito diretto dal latino medievale, che nel testamento serve per indicare due botteghe per la vendita di tessuti lasciate da Berrecci in eredità alle figlie. Si tratta di un latinismo medievale allora molto utilizzato in Polonia e in Boemia, soprattutto nei centri dove la vendita dei tessuti era di notevole importanza (p. es. spesso si ritrova negli Akty prawne di Cracovia). Il termine era diffuso con due significati: uno proprio nell' equivalente di 'bottega', l'altro nell'accezione di 'venditore', 'venditrice' (v. lemma 'Pannicida' in Słownik polszczyzny XVI wieku, IBL PAN, n.d.). Tale voce era di pubblico utilizzo anche nella lingua ceca. Infatti, in un dizionario di ceco antico all'interno del lemma 'budek' si nota proprio il sinonimo pannicida (Gebauer, 1903, p. 113). A volte il termine appare anche al neutro: Pannicidium. Interessante come Berrecci lo trasformi 
in un prestito integrato della sua lingua madre, accorpandogli il plurale maschile italiano in $-i$.

Nel testo sono presenti alcune forme del verbo essere al congiuntivo imperfetto: "l'uno ci fussi e che si fussino ambodua". Si tratta di forme del fiorentino quattrocentesco ancora presenti anche all'inizio del Cinquecento. Ciò trova conferma nell'evoluzione di Pietro Bembo: il fusse è, infatti, ancora presente nell'edizione del 1505 de Gli Asolani, dove, raggiunta un'ottima padronanza del fiorentino, il veneziano adegua la prosa ai tratti fonomorfologici propri della lingua fiorentina ed epura i tratti padani. La linea correttoria del Bembo che tornerà al fosse trecentesco al posto del fusse quattrocentesco ottiene conferma solo con l'edizione del 1530, quando già riesce nettamente a separare il fiorentino "aureo" del Trecento da quello "argenteo" del Quattrocento (Serianni, 2002, p. 211). Berrecci si trova nel bel mezzo di questa fase di passaggio, ma comunque geograficamente lontano, restando così in buona parte ancorato al modello fiorentino tardo quattrocentesco o primo Cinquecento.

Sempre riguardo alle forme verbali, si nota il passaggio di -ar protonica ad -er nel verbo essere: serà, ovvero il tipico - er fiorentino (Tavoni, 2015, p. 94), ma risulta presente l'estensione di -ar in un futuro dove ci sarebbe dovuto essere -er: trovarà. Si tratta qui di un caso antifiorentino, a conferma del fatto che l'artista portava con sé (giustamente) alcuni tratti linguistici acquisiti dal continuo contatto, tra l'altro all'estero, con altri toscani e italiani.

Continuando nella ricerca si osserva che Berrecci utilizza il toscano lasso per 'lascio', usato in alternanza già da Dante, ma che nel Quattrocento aveva decisamente preso il sopravvento. Un altro toscanismo è il verbo acconciare nell'accezione di 'sistemare', 'mettere a posto', presente già dal Trecento $\left(\mathrm{TLIO}^{10}\right.$ : voce 'acconciare').

Il pronome possessivo è qui declinabile, a differenza di un fiorentino dei primi del Cinquecento più puro (mia, sua) con pronomi indeclinabili, riscontrato per esempio nel citato trattato manoscritto Giuochi mathematici di Filicaia (Sosnowski, 2015, p. 61). Nel testamento si trovano, invece,

10 Tesoro della Lingua Italiana delle Origini consultabile on line sul sito web tlio. ovi.cnr.it/TLIO. 
sia il femminile mie - "le cose mie", "mie due figliole" - che il maschile miei, "li miei peccati". Non è presente l'articolo el che invece compare frequentemente nel testamento di Leonardo (Uzielli, 1872, pp. 202-210) (per maggior precisione si rammenta che l'arco temporale che intercorre tra i due documenti è di 17 anni). L'articolo el viene usato sistematicamente anche da Ariosto che lo sostituisce solo dopo il 1516 (Marazzini, 2002, p. 274). Non si riscontra il raddoppiamento fonosintatttico.

Conseguente alle dimensioni ridotte del documento è il numero non elevato dei modi, forme e tempi verbali inclusi nel testo, p. es. solo tre futuri mentre l'assenza del condizionale non permette un maggiore confronto con la morfologia verbale bembesca, punto forte dell'autore delle Prose. Si segnala anche la presenza dello scempiamento di alcune consonanti lunghe di origine settentrionale: citadino, anulando, legitimi, sepellito, avenire.

Sempre riguardo al lessico, nel testo si osserva la presenza del termine fiorentino beccaijo che Berrecci usa per localizzare una sua proprietà. Sul TLIO l'entrata 'beccaio' in questa forma nei secoli XIII-XIV si osserva solo nel fiorentino, rispetto ad altre forme come bechero (ven.), becharo (bol.), beché (mil.) e beccaro (sen.). Il latineggiante massaricie, invece, nel Quattrocento era popolare soprattutto nell'Italia settentrionale, utilizzato prevelentemente dal ceto mercantile. Curioso è il termine aureco con cui si localizza un'altra proprietà nella Judaica. Forse si intende un angolo della via a curva, a forma di orecchio, oppure dietro a un orefice ('aurefice' in TLIO), mestiere diffuso in quella via.

In ultimo si osservano alcuni termini polacchi che Berrecci prova a trascrivere, elementi che, sommati ad altri citati in precedenza, nel XIX secolo convinsero gli studiosi a definire "strana" la lingua utilizzata dal toscano (Sprawozdania Komisji..., Akademia Umiejętności, 1891, libro IV, p. XXVI).

Citando l'esecutore Malcher, Berrecci lo definisce podrenzi, con ogni probabilità dal titolo posseduto dall'amico, podrzędca (o podrzadca), cioè 'sostituto', 'vice', un tentativo di rendere dei termini polacchi diventati di uso comune anche nei documenti ufficiali latini.

Notevole è il "fu di Venzko" riferito alla casa dove Berrecci abitava. Il toscano utilizza il vezzeggiativo Venzko. Probabilmente il prece- 
dente proprietario dell'immobile si chiamava Wacław, che nel polacco medievale era Więcestaw (*vęteslavb in protoslavo). La versione tedesca era Wentzel, quella latina polonizzata Wenceslaus o Wenczeslaus (Kaczmarczyk, 1913, p. 5), dove la nasale $e$ si trasforma in nesso en. Berrecci usa dunque l'espressione Venzko, con il nesso en, proponendo oltre ad esso anche la $v$ latina (e volgare) al posto della $w$ polacca e l'addolcimento finale in $-k o$, tipico vezzeggiativo slavo, il che suggerisce un rapporto di conoscenza o amicizia.

Un altro immobile appartenente all'artista di Pontassieve era situato in via Viliska, il cui corrispondente sarebbe Wielicka, ovvero 'via per Wieliczka'. Il toponimo Wieliczka ha la sua etimologia nelle parole "Wielka sól”, 'grande sale' (Zejszner, 1843, p. 9). Lo storpiamento effettuato da Berrecci sembra presentare una certa naturalezza se si pensa alla lingua madre dell'autore, l'italiano, dove lo -cka [tska] finale dell'aggettivo spesso diventa, per motivi di difficoltà della pronuncia, $-s k a$, anche presente nella lingua polacca.

Continuando nella lettura del documento si nota la presenza di alcuni cognomi o soprannomi di altri proprietari di immobili, o meglio delle loro storpiature, espresse nell'intento di localizzare esattamente le proprietà elencate. Sempre riguardo a via Viliska prima compaiono Colassina e Urbanchova, poi Nogajska e Sluska. Nel primo accoppiamento è assai probabile che Berrecci si riferisse al terreno che nel Cinquecento apparteneva alla famiglia Kolasiński (corrispondente all'odierno numero 50 di via Krakowska) e di quello del commerciante Urban Rózyc (situato quasi di fronte al primo, oggi numero 47). Da qui il cognome della moglie, Kolasińska, storpiato in Colassina nel primo caso e l'appellativo Urbanchowa, ovvero 'moglie di Urban', nel secondo.

Sempre nella stessa via, in corrispondenza dell'odierno numero 51, nel XVI secolo si trovava Casa Nogawka, mentre in quello corrispondente all'odierno numero 53 vi era casa Służka, di Stanisław Służka. La proprietà di Berrecci, con il "fornaro", si trovava, dunque, in mezzo a queste due costruzioni ${ }^{11}$. Per le ricerche delle proprietà mi sono basa-

11 Fatto confermato anche da Estreicher che in un atto di vendita del 1542 ha riscontrato che l'immobile in questione viene segnalato tra casa Służka e casa Nogawka 
to soprattutto sugli studi di Krasnowolski (Krasnowolski, 1992, pp. 86, 88-89).

Interessante è dunque osservare come l'artista italiano, seppure con evidenti storpiature, si impegni, non senza difficoltà, nel cercare di rendere i cognomi degli allora proprietari che, probabilmente, conosceva personalmente. In tale contesto si cita ancora il curioso Anna caffeczanka. Assai complicato è arrivare con assoluta certezza al significato dell'appellativo caffeczanka, si possono solo fare delle congetture. La prima di esse è che si tratti semplicemente di un cognome (più correttamente Kawczanka). La seconda ipotesi è quella di una donna - in polacco la terminazione - anka è femminile - la cui famiglia proveniva da Caffa (nei secoli XIV e XV la presenza dei mercanti genovesi a Cracovia, arrivati sia dalla Superba che da Caffa, era fortemente attestata) ${ }^{12}$. Infine, potrebbe trattarsi di una trasposizione del termine fiorentino caffera, ovvero 'canfora', presente già dal Trecento $\left(\mathrm{OVI}^{13}\right.$ : voce 'caffera'). L'Anna in questione poteva, dunque, essere una commerciante di canfora.

In ultimo, come si è avuto modo di vedere, la città in cui l'autore visse è resa con la citata forma latina Casimiria, ma due volte anche con lo storpiamento Casmira; spesso nei documenti ufficiali appariva il prestito integrato Kasmir cui Berrecci pone caratteristiche italiane: la $c$ al posto della $k$ e la terminazione - $a$ finale.

$\mathrm{Nel}$ documento in questione si hanno, dunque, alcune testimonianze della lingua polacca di allora che lentamente si stava affrancando dal latino, proponendo, anche nei documenti ufficiali, termini propri o prestiti integrati.

(Estreicher, 1972, p. 49).

12 In effetti alcuni storici polacchi per definire gli abitanti di Caffa utilizzano il termine "Kaffańczycy", il cui femminile singolare poteva benissimo essere "Kaffańczanka" (vedi p. es. Małowist, 1947, pp. 50 e 314).

${ }_{13}$ Corpus OVI dell'italiano antico consultabile on line sul sito web gattoweb.ovi. cnrit. 


\section{CONCLUSIONI}

Il manoscritto del testamento di Berrecci necessitava di una nuova e più corretta trascrizione. Inoltre, dal punto di vista delle informazioni storiche fino ad oggi esso è stato analizzato solo parzialmente, mentre dal punto di vista linguistico era necessario un primo studio. Lo schema del testamento si è rivelato essere classico, diviso in tre parti. Si è confermato in buona parte l'utilizzo della lingua fiorentina di fine Quattrocento o inizi del Cinquecento, ma si riscontrano anche alcune influenze antifiorentine, frutto probabilmente di frequentazioni estere del Berrecci. Si tratta per lo più di una scrittura pratica, come dovrebbe essere, con uno stile vicino al colto, che offre alcune soluzioni stilistiche degne di nota, arricchito da forme latineggianti, da latinismi, tra cui uno forte a livello locale (pannicidi), e tentativi di inserimento di termini polacchi adattati in maniera più o meno coerente (p. es. viliska e Casmira) che dimostrano un tentativo da parte dell'autore di sentirsi parte integrante della comunità locale cui ha lasciato la sua forte impronta non solo artistica, ma anche sociale.

\section{BIBLIOGRAFIA}

Adamczewski, J. (1997). Mała Encyklopedia Krakowa. Kraków: Wanda.

Akademia Umiejętności (1891). Sprawozdania Komisji do Badania Historii

Sztuki w Polsce, index osobowy i rzeczowy do tomów I, II, III i IV, (vol.

IV). Kraków: Wydawnictwo Akademia Umiejętności

Akademia Umiejętności (1906). Sprawozdania z czynności i posiedzeń (voll.

11-15). Kraków: Nakładem Akademii Umiejęstności.

Cappelletti, L. (2011). Bartolomeo Berrecci da Pontassieve. Un genio del Rinascimento tra arte e filosofia. Firenze: Polistampa.

Estreicher, K. (1972). Szkice o Berreccim. Rocznik krakowski, XLIII, 45-115.

Fabiański, M. (2000). Il ruolo dell'arte italiana per l'architettura in Polonia. Studia italo-polonica, VI, 35-43.

Ferenc, M. (2012). Rachunki budowy zamku krakowskiego 1526. Kraków: Zamek Królewski na Wawelu. Państwowe Zbiory Sztuki.

Gaudioso, F. (1986). Testamento e devozione. Galatina: Congedo.

Gebauer, J. (1970). Slovník staročeský. Praha: Academia. 
IBL PAN (n.d.). Słownik polszczyzny XVI wieku. Retrieved from https://spxvi. edu.pl.

Kaczmarczyk, K. (1913). Libri iuris civilis cracoviensis 1392-1506. Kraków: Archiwum Akt Dawnych miasta Krakowa.

Kozakiewiczowa, H., \& Kozakiewicz, S. (1976). Renesans w Polsce. Warszawa: Arkady.

Łuszczkiewicz, W. (1879). Bartolomeo Berecci: architekt Kaplicy Zygmuntowskiej (-1537): oraz kilka wiadomości w przydatku do świeżej publikacyi p. t.: Album ozdób z Kaplicy Zygmuntowskiej i t. d. i t. d. Kraków: Nakł. Zarządu Muzeum Techniczno-Przemysłowego.

Małowist, M. (1947). Kaffa: kolonia genueńska na Krymie i problem wschodni w latach 1435-1475. Warszawa: Nakł. Tow. Miłośników Historii.

Mossakowski, S. (2007). Kaplica Zygmuntowska (1515-1533) Problematyka artystyczna i ideowa mauzoleum króla Zygmunta I. Warszawa: Liber pro Arte.

Olkiewicz, J. (1979). Opowieści o Włochach i Polakach. Warszawa: Ludowa Spółdzielnia Wydawnicza.

PAU (1935). Polski Słownik Biograficzny (vol. I). Kraków: Nakładem Polskiej Akademii Umiejętności

Pawlicki, B.M. (2015). Związki polsko-włoskie w architekturze doby odrodzenia 1500-1600. Teka komisji i architektury PAN Oddziat w Krakowie, XLIII, 19-39.

Pelc, J. (2000). Barok w Polsce i w Europie Środkowo-Wschodniej. Warszawa: Wydział Polonistyki, Instytut literatury polskiej.

Ptaśnik, J. (1909). Gli italiani a Cracovia. Roma: Forzani e C..

Serianni, L. (Ed.). (2002). La lingua nella storia d'Italia. Roma: Scheiwiller/ Società Dante Alighieri.

Sosnowski, R. (2015). Tra Firenze e Borgo San Sepolcro. Una ricognizione sulla lingua del testo inedito di Giuochi mathematici di Piero da Filicaia. In E. Jamrozik \& R. Sosnowski (Eds.), Percorsi linguistici tra Italia e Polonia, studi di linguistica italiana offerti a Stanisław Widłak (pp. 55-63). Firenze: Franco Cesati.

Szelińska, W. (1990). Książka Erazma z Rotterdamu w środowisku krakowskim $W X V I$ wieku. Kraków: WSP.

Tavoni, M. (2015). Storia della lingua italiana. Il quattrocento. Bologna: il Mulino.

Uzielli, G. (1872). Ricerche intorno a Leonardo da Vinci. Firenze: Stabilimento di G. Pellas. 
Zejszner, L. (1843). Krótki opis historyczny, geologiczny i górniczy Wieliczki. Berlin: Nakładem B. Behra.

\section{MANOSCRITTI}

Acta d. scabinorum civitatis Kazimirie pos exustionem pretorii per manus Bartolomei Cromeri notarii comperata (1536), Archiwum miasta Kazimierz pod Krakowem, Dokumentacja aktowa, tomo 380, pp. 364 e 365, catalogato in: Księgi ławnicze główne (indukty). Inskrypcje i sprawy sporne (inscriptiones, controversiae)

Acta officialia Archidiecezji Krakowskiej (1520-1522), tomo 57.

Filicaia, P. da (1516). Giuochi mathematici, Collezione manoscritti berlinesi, manoscritto Ms. ital. Quart. 48. Biblioteca Jagellonica di Cracovia.

Riassunto: Il seguente articolo presenta l'analisi del testamento del più famoso scultore e architetto del Rinascimento in Polonia: il toscano Bartolomeo Berrecci, autore, tra le altre opere, della Cappella di Sigismondo nella cattedrale del Wawel a Cracovia. Dopo una breve introduzione storico-culturale in cui vengono presentati il suo arrivo in Polonia e la sua funzione alla corte del re Sigismondo il Vecchio, viene riportata la trascrizione del testamento dell'artista, redatto di propria mano in lingua italiana e conservato negli archivi dell'antica città di Casimiria, cui segue un'approfondita analisi. Dapprima essa sarà di carattere contenutistico e strutturale: verranno infatti esposti i personaggi e le proprietà citate nel documento, così come la sua forma. In seguito si passerà alle analisi morfologica e terminologica in cui si troveranno conferme dell'utilizzo della lingua fiorentina di fine Quattrocento e primi Cinquecento, ma anche la presenza di termini specifici, come p. es. prestiti integrati, legati alla realtà polacca in cui l'artista visse per quasi un ventennio, conferendo al documento stesso, oltre ai classici valori spirituale e notarile, anche quelli storico e linguistico.

Parole chiave: lingua italiana nel Cinquecento, Bartolomeo Berrecci, testamento di Bartolomeo Berrecci, arte rinascimentale, Rinascimento a Cracovia 